\title{
Chromospheric models of a solar flare including velocity fields
}

\author{
A. Falchi ${ }^{1}$ and P. J. D. Mauas ${ }^{2}$ \\ 1 Osservatorio Astrofisico di Arcetri, 50125 Firenze, Italy \\ 2 Instituto de Astronomía y Física del Espacio, Buenos Aires, Argentina \\ e-mail: pablo@iafe.uba.ar
}

Received 14 December 2001 / Accepted 25 March 2002

\begin{abstract}
We study the chromospheric structure of a small flare, before, during and after the first hard X-ray spike. We construct 5 semiempirical models for different times, which will reproduce the profiles of the $\mathrm{H}_{\delta}$, Ca II K and Si I 3905 lines during the flare evolution. In order to reproduce the intensity and the main characteristics of the line asymmetry, we introduce the velocity fields in the profile calculations. We find that the whole chromosphere undergoes a strong upward motion within $1 \mathrm{~s}$ of the maximum of the first hard X-ray spike, when the temperature and the density begin to rapidly increase. This seems the first chromospheric response to the energy deposition and/or release. Only $6 \mathrm{~s}$ after the hard X-ray peak, a strong downflow begins in the low chromosphere and its velocity continues to increase even during the cooling phase.
\end{abstract}

Key words. Sun: chromosphere - Sun: flares

\section{Introduction}

During the impulsive phase of a flare the chromosphere might be heated by non-thermal electron impact (thick target model) and/or by thermal conduction from the heated corona. For both energy deposition mechanisms a strong evaporation of chromospheric plasma drives a downward motion of a chromospheric condensation (Fisher 1985; Gan et al. 1991; Abbett \& Hawley 1999). A red-shifted emission of chromospheric lines, observed during the impulsive phase of flares (Ichimoto \& Kurokawa 1984), is considered a signature of such a condensation motion. For a comprehensive review of the observational scenario on dynamics of flares we refer to Falchi (2002) and Heinzel et al. (1994).

The duration and temporal dependence of the downflow related to the chromospheric condensation have been studied by Fisher (1989). He found that the duration of the downflow depends on the pressure scale-height of the preflare chromosphere, while its maximum speed depends both on the energy flux driving the chromospheric evaporation and on the preflare chromospheric density. Therefore, the study of the velocity pattern, before and during the impulsive phase of a flare, together with the chromospheric preflare atmosphere, can be an important issue to probe the flare energetics.

Send offprint requests to: A. Falchi,

e-mail: falchi@arcetri.astro.it
The line-of-sight velocity is often determined by simply interpreting the wavelength shift $\Delta \lambda$ of the observed line profile asymmetry (defined generally with the bisector) in terms of Doppler shift. One has to be very careful for optically thick lines (as for example $\mathrm{H}_{\alpha}$ ), since this method can result in an incorrect estimate of the magnitude of the velocity (Athay 1976; Beckers 1981) and can even limit the possibility of recovering the direction of the velocity (Gan et al. 1993; Heinzel et al. 1994).

It has also been observed that the shift of the bisector with respect to the undisturbed central wavelength of the line, increases from the center towards the wings (Falchi et al. 1992; Wülser et al. 1994). This fact has been interpreted as evidence of a depth dependence of the velocity, which is not consistent with the usual assumptions of the simulations. Therefore, to clarify the observational framework of line asymmetries, computed ad hoc atmospheric models are needed to determine the velocity and its depth dependence.

The aim of this paper is to construct semiempirical models matching the $\mathrm{H}_{\delta}$, Ca II K and Si I $\lambda 3905 \AA$ line profiles, at several times during a flare observed on June 7 , 1991. The models were computed considering a depth dependent velocity to match the asymmetry of the 3 lines.

In Sect. 2 we present the observations and the flare characteristics. In Sect. 3 we explain how we built our models, which are presented in Sect. 4, together with the computed and observed profiles. In Sect. 5 we describe the 

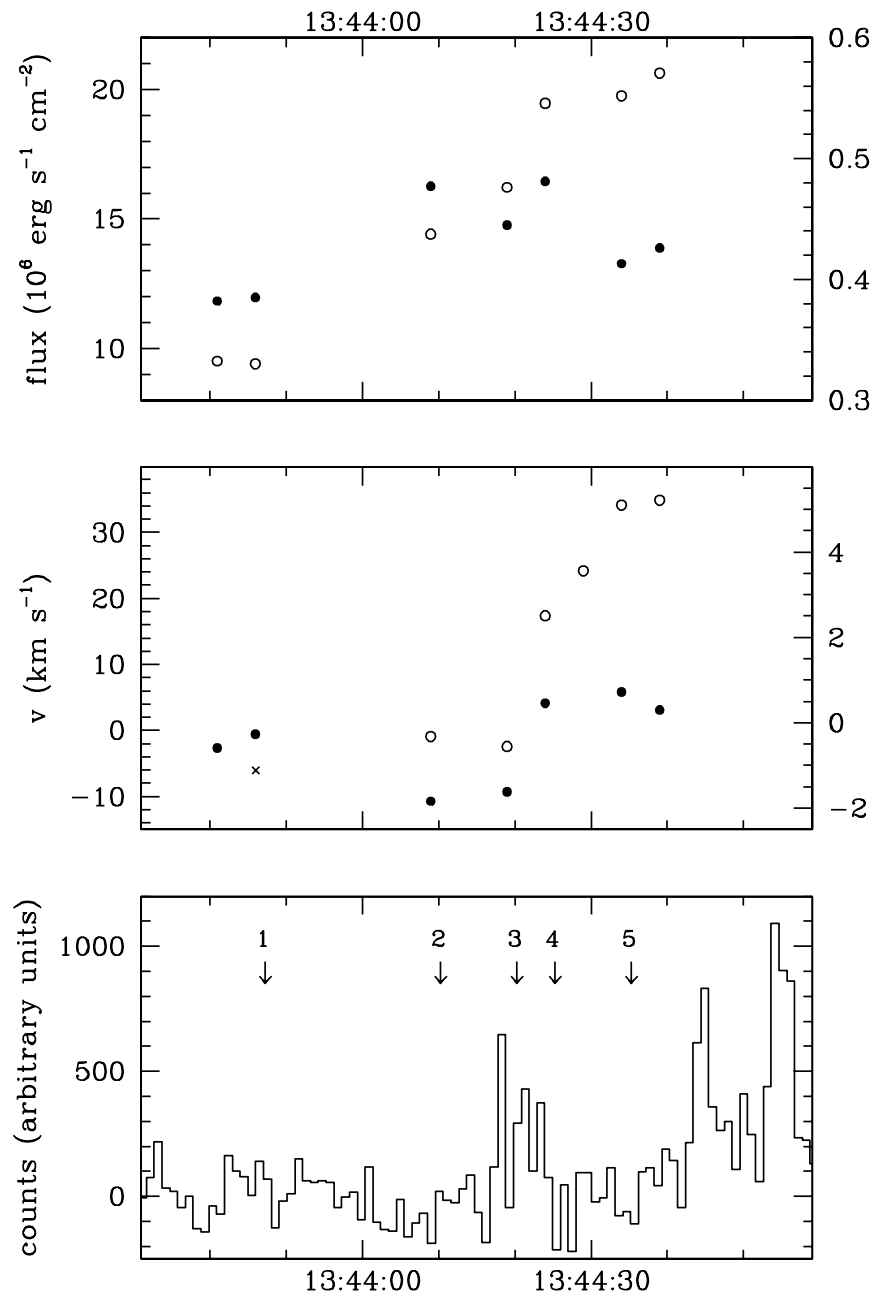

Fig. 1. Temporal evolution of the flare. Top panel: net flare emission integrated over the lines Ca II K (open circles) and Si I (filled circles) in units of $10^{6} \mathrm{erg} \mathrm{cm}^{-2} \mathrm{~s}^{-1}$; Middle panel: velocity determined from Ca II K (the asterisk indicates the velocity from the shift of its self-absorption) and Si I; a negative value indicates an upward velocity. For both panels the $y$-scale on the left refers to Ca line and the $y$-scale on the right refers to the Si line. Bottom panel: hard X-ray light curve in arbitrary units, as detected by the BATSE instrument on CGRO, in the energy range $25-50 \mathrm{keV}$. The arrows indicate the times for which we compute the models.

temporal evolution of the chromosphere during the flare, and in Sect. 6 we discuss the results.

\section{The observations and the flare characteristics}

A small flare, developed in AR NOAA 6659 on June 7, 1991, was observed during a coordinated observing campaign. A detailed description of the program is given in Cauzzi et al. (1995).

The optical spectra were obtained at the Vacuum Tower Telescope of the National Solar Observatory at Sacramento Peak with the Universal Spectrograph (USG), in the range $3500-4200 \AA(\delta=1.5 \AA / \mathrm{mm})$. The seeing quality during our observations was very high $\left(0.5^{\prime \prime}\right)$. The spectra were acquired with a temporal resolution of
$5 \mathrm{~s}$ at the beginning of the flare and of $20 \mathrm{~s}$ in the later phase, and have been digitized with a scale of $0.68^{\prime \prime}$ per pixel. The HXR data, in the energy range $25-100 \mathrm{keV}$, are from the BATSE instrument on the Compton Gamma Ray Observatory (CGRO).

The flare developed in the penumbra of a large spot. The emission properties and the velocity field of this flare have been analyzed in Cauzzi et al. (1995, 1996). We remind here two of its most important characteristics:

- At least $40 \mathrm{~s}$ before any observed HXR emission, the kernel shows a typical chromospheric flare spectrum. The Balmer lines are resolved up to $\mathrm{H}_{13}$; the $\mathrm{Ca}$ II $\mathrm{H}$ and $\mathrm{K}$ lines have a strong emission core, as well as several other metallic lines (e.g. Fe I, Si I, Al I and Mg I).

- The flare hard X-ray (HXR) emission, in the range 25-100 keV, consists of five separate and short spikes (lasting 1-7 s), without interspike emission. This simple timedependent structure suggests that this flare is a sequence of separate elementary bursts.

In this paper we concentrate on one small region of the flare, of about $1^{\prime \prime}$, (within the kernel B of Cauzzi et al. 1997, hereafter CFFS), which is the one that shows the largest emission and the strongest velocity effects. We study its evolution before, during and after the first HXR spike (from 13:43:46 UT up to 13:44:34 UT), and we build five models of the atmosphere as it changes during the flare, with particular attention to the variations of the velocity field.

We consider the net flare emission, i.e. we subtract the spectrum of the "quiet" penumbra, averaged over various points close to the kernel area, from the total spectrum of the flare. In Fig. 1 we show the temporal evolution of the net flare emission for this region, integrated over the Ca II K and Si I 3905 lines, together with the velocities obtained by CFFS from the two line profiles. We do not show $\mathrm{H}_{\delta}$ or other metallic lines because their behavior is similar to Ca II K and to Si I, respectively. The hard X-ray emission is also shown in the bottom panel. During the flare the measured line emission increases almost linearly with time while the velocity changes its sign (from upward to downward) with the first HXR spike and continues to increase for at least $10 \mathrm{~s}$ after the end of the spike.

We briefly recall the different methods used to derive the velocity from the two lines. Since the Ca II K line (as well as $\mathrm{H}_{\delta}$ ) shows a strong asymmetry, to derive the velocity CFFS computed the bisector of the emission profile excluding, if present, the central self aborption. The shift of the bisector from the wavelength at rest is not constant within the profile, but increases from the line centre towards the wings. Interpreting the shift of the bisector in terms of Doppler shift, CFFS computed a velocity which is not constant within the layers contributing to the emission. We remark that the minimum of the selfabsorption, when present, shows a blue-shift corresponding to an upward velocity (here indicated as negative) of about $-6 \mathrm{~km} \mathrm{~s}^{-1}$. The velocity plotted in Fig. 1 is the one corresponding to the maximum shift in the wings and 
Table 1. Characteristics of the models. We give in Col. 1 the time of the observed spectra corresponding to our computed models, in Col. 2 the peak time for the first HXR spike, and in the following columns the hydrogen density, the column mass and the temperature at $1100 \mathrm{~km}$ (measured from the point where $\tau_{5000}=1$ ). In the last column we give the coronal pressure.

\begin{tabular}{|c|c|c|c|c|c|}
\hline Time & Peak time & $\mathrm{NH}$ & $m$ & $T$ & $P_{\text {cor }}$ \\
\hline $\mathrm{UT}$ & UT & $10^{13} \mathrm{~cm}^{-3}$ & $10^{-2} \mathrm{~g} \mathrm{~cm}^{-2}$ & $\mathrm{~K}$ & dyn $\mathrm{cm}^{-2}$ \\
\hline $113: 43: 46$ & & 6.29 & 3.18 & 7480 & 12.98 \\
\hline 2 13:44:09 & & 6.63 & 3.55 & 7782 & 19.17 \\
\hline 3 13:44:19 & $13: 44: 18$ & 6.59 & 3.86 & 8164 & 24.59 \\
\hline 4 13:44:24 & & 7.07 & 4.30 & 8349 & 31.81 \\
\hline 5 13:44:34 & & 6.60 & 3.86 & 8164 & 28.08 \\
\hline
\end{tabular}

hence refers to the lower chromospheric layers and not to the higher ones contributing to the central self-absorption.

The Si I 3905 line, on the other hand, does not show any asymmetry of the emission profile, but its emission peak is shifted with respect to the unperturbed wavelength. This shift is interpreted by CFFS as a global Doppler shift.

\section{The models}

To study the evolution of the flare, in this paper we concentrate in the chromospheric effects of the first HXR spike, which reached its maximum intensity at 13:44:18 UT and lasted $7 \mathrm{~s}$. To do so, we build semiempirical models of the flare atmosphere at 5 different times, shown by the arrows in Fig. 1, and listed in Table 1. From now on, we will refer to each spectra by the number given in this table. Therefore, we modelled the atmosphere for the spectra taken at two times before this spike (models 1 and 2), for one spectrum taken at the beginning of the spike (model 3), and two spectra obtained after the spike (models 4 and 5 ).

The modelling was done using the program Pandora (Avrett \& Loeser 1984). Given a $T$ vs. $z$ distribution, we solved the non-LTE radiative transfer, and the statistical and hydrostatic equilibrium equations, and selfconsistently computed non-LTE populations for 10 levels of H, 13 of He I, 9 of C I, 15 of Fe I, 8 of Si I, Ca I and Na I, 6 of $\mathrm{Al} \mathrm{I}$, and 7 of $\mathrm{Mg} \mathrm{I}$. In addition, we computed 6 levels of He II and Mg II, and 5 of Ca II. For every species under consideration we include all the bound-free transitions and the most important bound-bound transitions. Ly- $\alpha$, the $\mathrm{Ca}$ II $\mathrm{H}$ and $\mathrm{K}$ and $\mathrm{Mg}$ II $\mathrm{h}$ and $\mathrm{k}$ lines where all computed with a full partial redistribution treatment. For the hydrogen atom we use the collisional rates of Johnson (1972), for all transitions except Ly- $\alpha$, for which we used the results by Scholz et al. (1990), and $\mathrm{H}_{\alpha}$ and $\mathrm{Ly}-\beta$, for which we used the results by Giovanardi et al. (1987) and Giovanardi \& Palla (1989). The Einstein coefficients were also taken from Johnson (1972) and the photoionization cross-sections are from Mathisen (1984). The remaining parameters were considered as it is explained in Vernazza et al. (1981).

For the Ca II K and $\mathrm{H}$ lines we used the Einstein coefficients of Black et al. (1972), and the Van der Waals and Stark broadening coefficients by Monteiro et al. (1988) and Konjevic et al. (1984) respectively. For the collisional rates for these transitions, we had to scale the values by Taylor \& Dunn (1973) by a factor of 5 (that is, a factor of 1.5 with respect to the ones of Shine \& Linsky 1974), since the published values result in a central intensity much lower than observed. A similar problem was already found in Mauas \& Falchi (1994), who could not reproduce the $\mathrm{H}_{\gamma}$ and the Ca II K intensity simultaneously.

The Si I line formation was studied by Cincunegui \& Mauas (2001), and here we use the same atomic model. As pointed out in that paper, the irradiation of the low chromosphere by ultraviolet lines originating in the upper chromosphere or the transition region can strongly affect the ionization balance of Si I. To take this effect into account, in this paper we use the line list given by Cincunegui \& Mauas (2001) (their Table 4). We found that, to match the observed intensity of the line at $3905 \AA$, we need a flux of about $5 \times 10^{7} \mathrm{erg} \mathrm{cm}^{-2} \mathrm{~s}^{-1}$ integrated over the range 1200-1600 $\AA$, in good agreement with flare values found by Brekke et al. (1996).

Several studies on the effect of electron beams on the flare emission have dealt with the significance of nonthermal collisional excitation and ionization rates on the line profiles of hydrogen and Ca II K (e.g. Fang et al. 1993; Kasparová \& Heinzel 2002). We estimated these rates for the main transitions considered here, following the method developed by Gómez \& Mauas (1992) and Mauas \& Gómez (1997) and found that they are much smaller than the thermal rates for this particular flare. The energy flux of electrons above $20 \mathrm{keV}$ estimated in CFFS is about $4 \times 10^{8} \mathrm{erg} \mathrm{cm}^{-2} \mathrm{~s}^{-1}$, a factor 25 smaller than the smallest value considered, for example, by Kasparová \& Heinzel (2002).

As a first order approximation, we considered a temperature distribution and computed the continuum intensity and the emerging profiles for the three lines under study, and compared them with the observations. We then modified the assumed $T$ vs. $z$ until a satisfactory match between observations and calculations was obtained, neglecting the observed line asymmetries. A microturbulence of $3 \mathrm{~km} \mathrm{~s}^{-1}$ was assumed throughout the chromosphere and no other broadening mechanisms such as macroturbulence were considered. In a second step we introduced a $v$ vs. $z$ distribution, and self-consistently recomputed the radiative transfer and statistical equilibrium. We then modified the $v$ vs. $z$ distribution until we obtained a satisfactory match with the line asymmetries. The velocity fields, however, were not considered to compute the pressure structure of the atmosphere, and the results obtained from the hydrostatic equilibrium without the velocity were left unchanged.

Before the HXR spike, the observed asymmetries are small, and therefore the velocities we found are very low. 

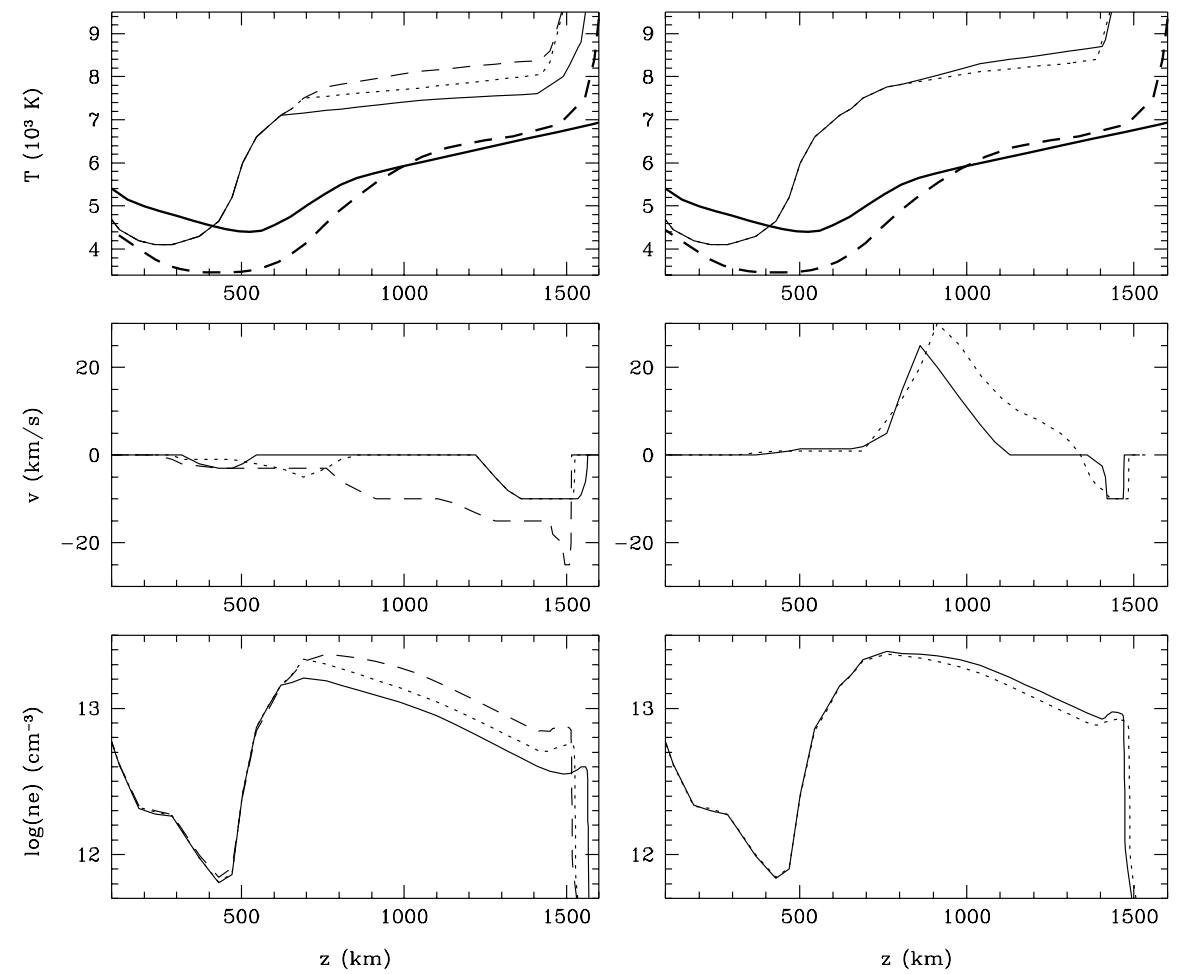

Fig. 2. The computed atmospheric models. The panels at left show the models for times 1 (full-line), 2 (dotted line) and 3 (dashed-line), before and at the beginning of the first X-ray spike, and the panels at right show the models for times 4 (full-line) and 5 (dotted line), after the first X-ray spike. Top panels: temperature vs. height distributions. The quiet-Sun model C (VAL81) and the penumbral model by Ding \& Fang (1989) are shown for reference (thick line, full and dashed respectively). Mid panel: velocity distributions; a negative value indicates an upward velocity. Bottom panel: electron density.

In this case, we test the models including the velocity field in the hydrostatic equilibrium equations, and found that the effect on the profiles of the considered lines was negligible. This was expected, since the contribution to the total pressure of the macroscopic velocity is negligible with respect to that of the thermal and turbulent velocities. During or immediately after the HXR spike, the asymmetries are much stronger and therefore the derived velocities are much larger. In this case, what would be needed is to self-consistently solve the Euler and the continuity equations, together with the radiative transfer and statistical equilibrium equations, a task beyond the scope of this paper. However, the heating process, due to some burst of energy release, should occur on a time scale much shorter than the hydrodynamic time scale. For example, Canfield \& Gayley (1987) estimated that the heating time scale by a beam of relativistic electrons is of about $0.05 \mathrm{~s}$, while the hydrodynamic effect of this rapid heating would be effective on a time scale of about $5-10 \mathrm{~s}$. We therefore feel that the two effects can effectively be separated, and that the models presented here can be considered as a good first order approximation to the real situation.

\section{Results}

Since the flare developed in a sunspot penumbra, we could not use the photospheric temperature distribution of the quiet Sun, and had to modify the temperature at these levels to match the observed emission between 3600 and $4100 \AA$. We point out that in this spectral range there are no "true continuum" windows, and we had to use the highest intensity points of the observed emission to construct the model at photospheric levels.

At chromospheric levels the models were constructed to match the line profiles of the $\mathrm{Ca}$ II $\mathrm{K}$ line, $\mathrm{H}_{\delta}$ and Si I 3905. The Ca II $\mathrm{H}$ line and $\mathrm{H}_{\epsilon}$, which are the other strong lines in the observed spectral region, are not used since they have more or less the same formation regime. Also, since these two lines are blended they are much more difficult to interpret. We notice that the blue $\mathrm{K}_{1}$ minimum of the Ca II $\mathrm{K}$ line is a very important signature to constrain the region of the temperature minimum and of the onset of the chromospheric rise.

In Fig. 2 we show the distribution of the temperature $T$, of the electron density $n e$ and of the velocity $v$ as a function of height in the atmosphere, measured from the point where $\tau_{5000}=1$. The models constructed for the 5 considered times are shown together with the quiet Sun model C from Vernazza et al. (1981) (VAL81) and the penumbral model by Ding \& Fang (1989), for reference. The main characteristics of each model are listed in Table 1. Of course, there is an indetermination in the models, in the sense that each model is not the unique possible atmospheric structure that emits the observed features. However, in order to match the observed line profiles, the allowed temperature and electron density variations are 

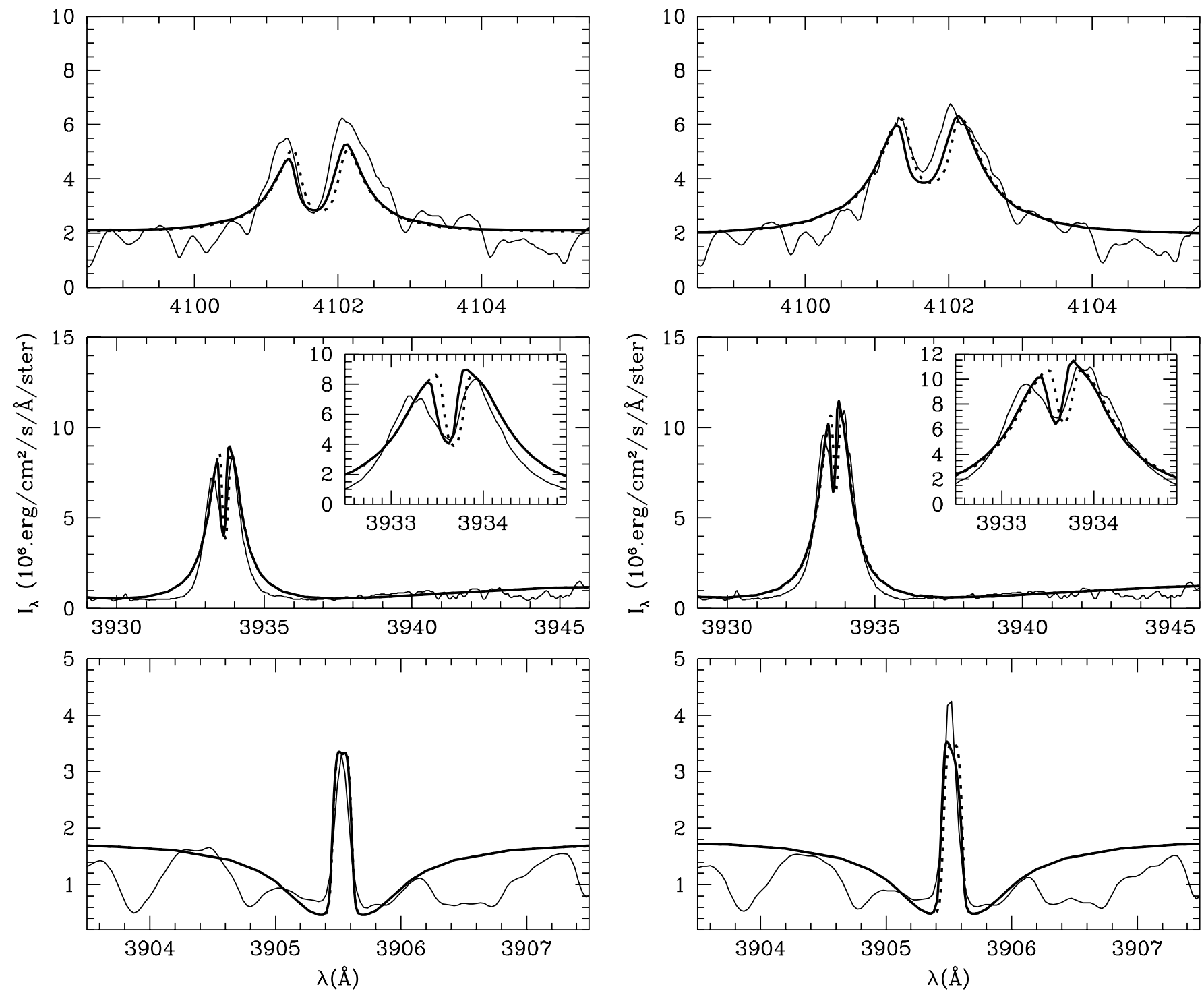

Fig. 3. Observed (thin line) and computed (thick line) profiles for $\mathrm{H}_{\delta}$ (top panel), Ca II K (middle panel), and the Si I line at $3905 \AA$, for the model 1 (13:43:46 UT). Also shown are the profiles computed for the same $T$ vs. $z$ distribution, but without velocity fields (dotted line).

relatively small. To prove this point, we performed a very large number of trials that all gave consistent results. We are, therefore, confident that the order of magnitude and the sign of the velocity we found are reliable.

Figures 3 to 7 show the observed and computed line profiles at each considered time. For each line we also show the profile that would be obtained for the same $T$ vs. $z$ distribution, but with no velocity field (i.e. symmetric). It can be seen that the general agreement between the observed and the computed profiles is fairly good for all times: the absorption wings of the Ca II and of the Si I lines are well matched as well as the peak intensity of the three lines.

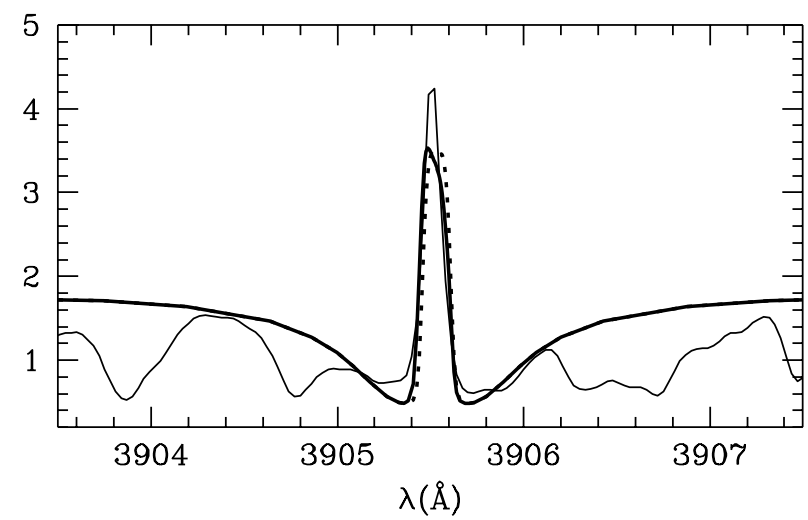

Fig. 4. Model 2 (13:44:09 UT); symbols are as in Fig. 3.

\section{Time dependence of the atmospheric parameters}

During the flare the atmosphere changes only above $600 \mathrm{~km}$ (measured from the point where $\tau_{5000}=1$ ), while the layers from the photosphere to the low chromosphere remain unchanged. This reflects two important observed features during the considered time interval: on one hand, the emission in the windows of the highest intensity is constant within the photometric precision limit of about $5 \%$ and no Balmer continuum emission was detected in any of the spectra; on the other, the intensity in the blue $\mathrm{K}_{1}$ minimum of the Ca II K line is constant within $10 \%$.

The fact that this flare has the chromospheric lines in emission well before any observed HXR, translates into a model with the chromospheric temperature and density higher than the ones of the quiet Sun, already $22 \mathrm{~s}$ before the first HXR spike. This indicates that some kind of energy release and/or energy deposition has already been in place. 

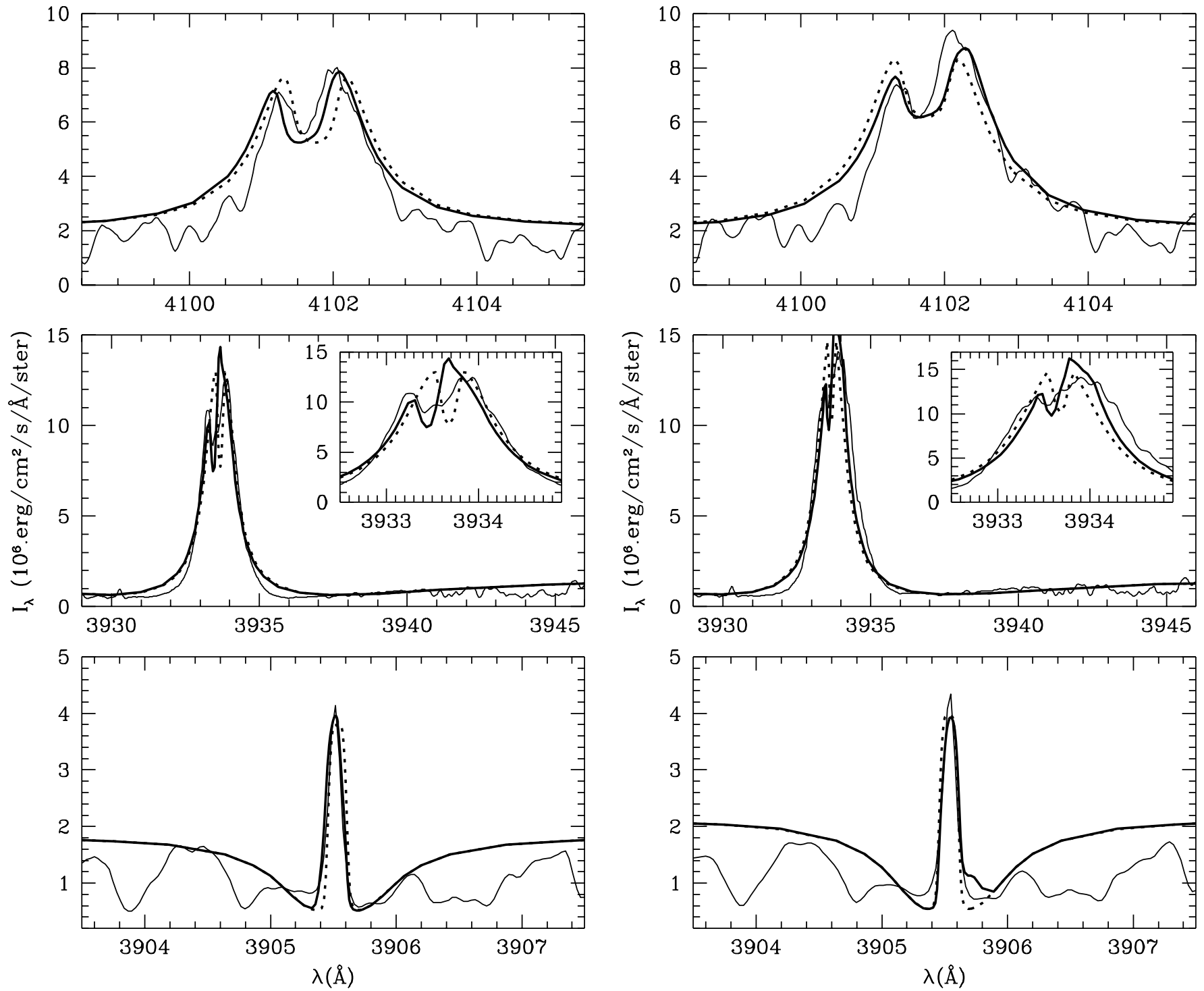

Fig. 5. Model 3 (13:44:19 UT); symbols are as in Fig. 3.

To better illustrate how the atmosphere changes with time we show in Fig. 8 the time dependence of the temperature and the electron density at a low chromospheric level (height $\approx 900 \mathrm{~km}$ ) and at a high chromospheric level (height $\approx 1400 \mathrm{~km}$ ).

We see that the chromospheric temperature begins to increase before any detectable HXR emission, at first with a small gradient and later with a higher gradient, reaching the peak value at 13:44:24 UT. A similar behaviour is shown by the electron density. Both the temperature and the electron density of the flare chromosphere peak $6 \mathrm{~s}$ after the peak time of the HXR burst and decrease afterwards, reaching at 13:44:34 UT values similar to the ones of 13:44:19 UT. This implies that, whatever the mechanism responsible for the pre-flare heating, it is still at work at least until the time of model 2 , while later on the dominant mechanism of energy deposition should be related to the HXR spike.

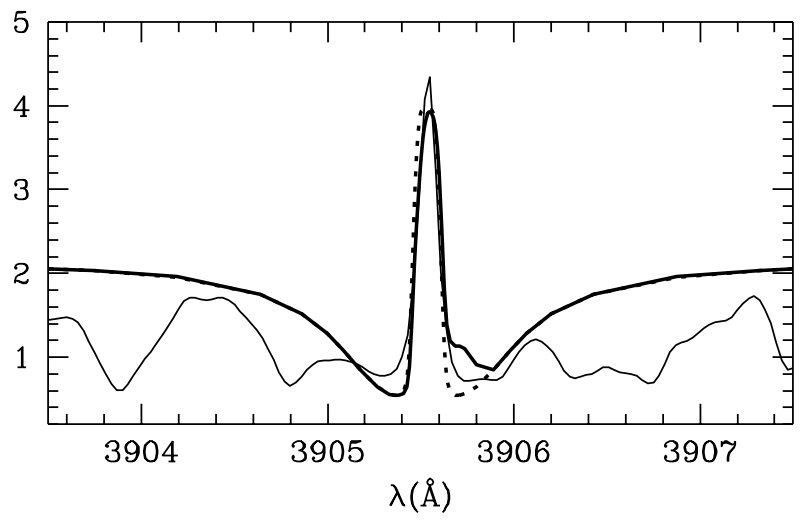

Fig. 6. Model 4 (13:44:24 UT); symbols are as in Fig. 3.

The coronal pressure also increases from time 1 up to time 4 (see Fig. 8), and the transition region moves downward. This is to match the observed self-absorption of the $\mathrm{Ca}$ II and $\mathrm{H}_{\delta}$ line profiles, which decreases from time 1 to time 4. A decreasing self-reversal due to an increasing coronal pressure has been already illustrated by Canfield et al. (1984) and Falchi et al. (1990) for $\mathrm{H}_{\alpha}$ and $\mathrm{H}_{\delta}$, respectively. The coronal pressure peaks simultaneously with the chromospheric temperature and density and decreases afterwards.

Before the spikes, at times 1 and 2, by matching the small observed asymmetry in the profiles we found a velocity directed upward in two distinct regions of the atmosphere (see Fig. 2). To match the asymmetry in the $\mathrm{H}_{\delta}$ and Ca II K peaks (the red peak is higher than the blue one), we needed to move the central absorption for both lines blueward, introducing an upward velocity of $-10 \mathrm{~km} \mathrm{~s}^{-1}$ in the high chromosphere (see Heinzel et al. 1994 for a similar effect). Note that the red asymmetry observed in 

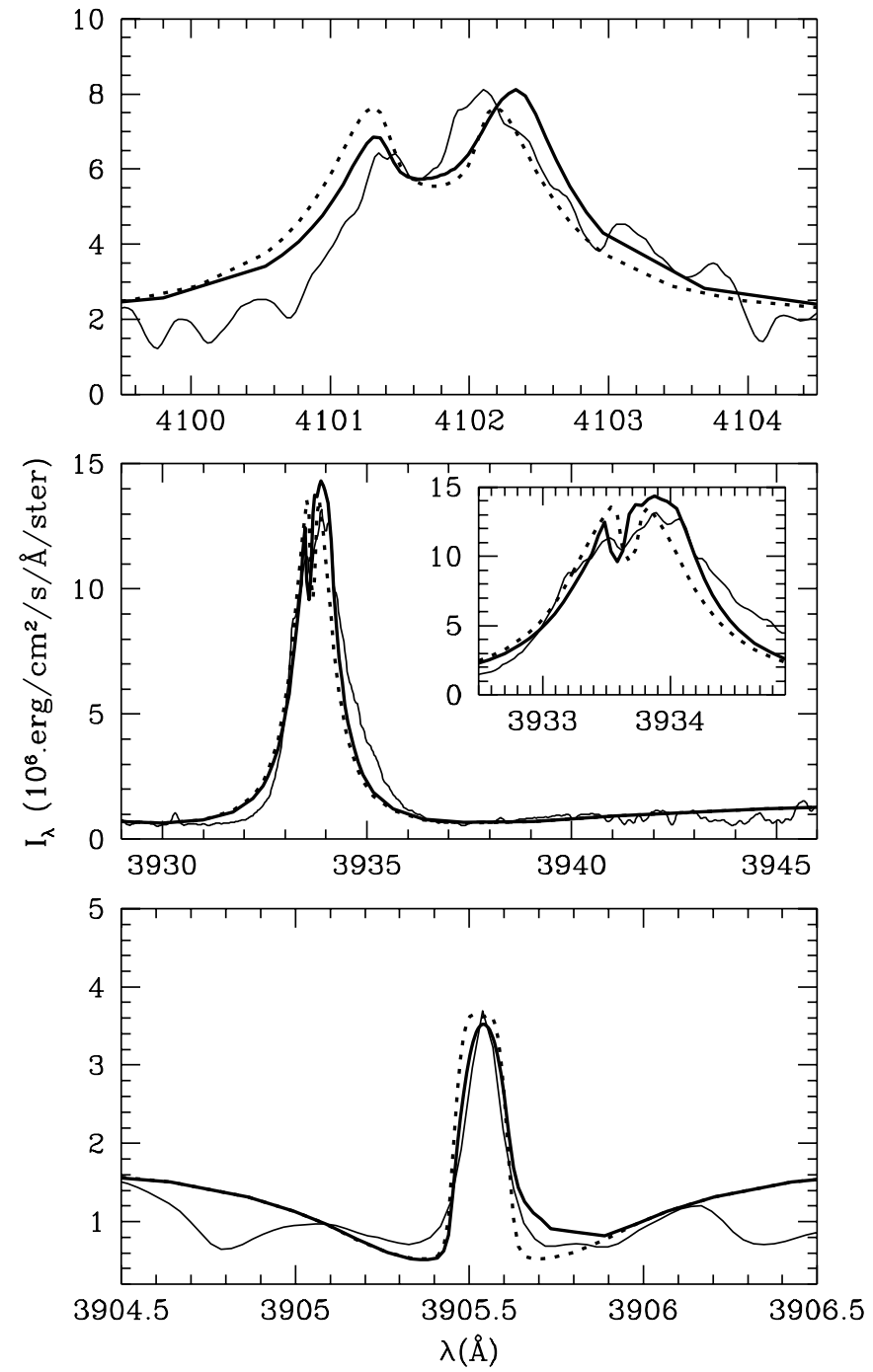

Fig. 7. Model 5 (13:44:34 UT); symbols are as in Fig. 3.

the line peaks comes from this upward motion of the upper part of the atmosphere, and not from a downward motion of the regions where this part of the line is formed. On the other hand, the Si line is displaced blueward from its wavelength at rest, and we therefore had to include an upward velocity in the Si line formation region, although in this case of only -3 to $-5 \mathrm{~km} \mathrm{~s}^{-1}$.

At the time of model 3, within $1 \mathrm{~s}$ of the first HXR spike, we found a velocity directed upward in the whole atmosphere, with a magnitude increasing from the low chromosphere up to the transition region, reaching $-30 \mathrm{~km} \mathrm{~s}^{-1}$ (see Fig. 2). This important characteristic of the atmosphere is not readily visible in the measurements of Fig. 1, but is clearly outlined by the accurate modelling. Such a rising motion might be an important signature of the first energy release and/or deposition at chromospheric levels.

After the spike, the velocity in the high chromosphere (at the boundary with the transition region) remains of the same order than before, but in the lower atmosphere becomes directed downward and changes with height, reaching $35 \mathrm{~km} \mathrm{~s}^{-1}$ at $h=900 \mathrm{~km}$. This is necessary to simultaneously reproduce the blue-shift of the selfabsorption and the strong red asymmetry observed in the wings of $\mathrm{H}_{\delta}$ and $\mathrm{Ca}$ II $\mathrm{K}$. However, altough we can match the asymmetry observed in the central part of the emission profiles, we were not able to match the strong asymmetry seen in the far emission wings of the $\mathrm{Ca} \mathrm{K}$ and $\mathrm{H}_{\delta}$ lines, in particular for the last model, as shown in Fig. 7.

To reproduce this feature, we would have to include very large velocities in the layers just above the temperature minimum, where this part of the profiles are formed. However, this is the region where the Si line is formed, and in this case the observed global shift is much smaller, and is reproduced quite well with the included velocity.

The behaviour of the chromospheric velocity is summarized in Fig. 8 for two chromospheric layers $(h \approx 1400 \mathrm{~km}$ and $h \approx 900 \mathrm{~km})$. We see very clearly that the whole atmosphere undergoes a strong upward motion, within $1 \mathrm{~s}$ of the maximum of the first HXR spike, when the temperature and the density begin to increase with a higher gradient. Only afterwards, a strong downflow begins in the low chromosphere and continues to increase even when the entire atmosphere begins to settle back. A similar result was mentioned in a review of previous observations by Švestka (1976), who reports that a blue asymmetry is seen at the onset of $23 \%$ of the observed flares. This asymmetry disappears within one minute, and afterward a strong red asymmetry sets in. In more recent observations (e.g. Canfield et al. 1990) a blue asymmetry of the $\mathrm{H}_{\alpha}$ line has been found only sporadically and confined in small structures that did not present a strong red asymmetry afterward.

We notice here that the magnitude and the direction of the velocity inferred by the models are fairly consistent with the values measured by CFFS using the bisector method in the Ca II wings. As mentioned in Sect. 2 the velocity in the higher layers must instead be recovered using the shift of the central self-absorption.

\section{Discussion}

We have presented models for 5 different moments during the evolution of a flare, before, simultaneously, and after the first observed X-ray spike. The models refer to a very small area $\left(1^{\prime \prime}\right)$ of the flaring kernel and to a very short time interval $(\approx 50 \mathrm{~s})$ at the beginning of the flaring episode. We reproduce the observed profiles of the Ca II K, $\mathrm{H}_{\delta}$ and the Si I $3905 \AA$ lines.

We included an adequate velocity field in the profile calculations, although not in the atmospheric dynamics. With this velocity field that varies with height in the atmosphere we were able to match not only the mean profiles but also the main characteristics of the observed asymmetries.

We find that the flare only affects the atmospheric temperature above $600 \mathrm{~km}$, and that both the temperature and the density begin to increase before any detectable HXR emission, and peak $6 \mathrm{~s}$ after the first peak of the X-ray burst. 

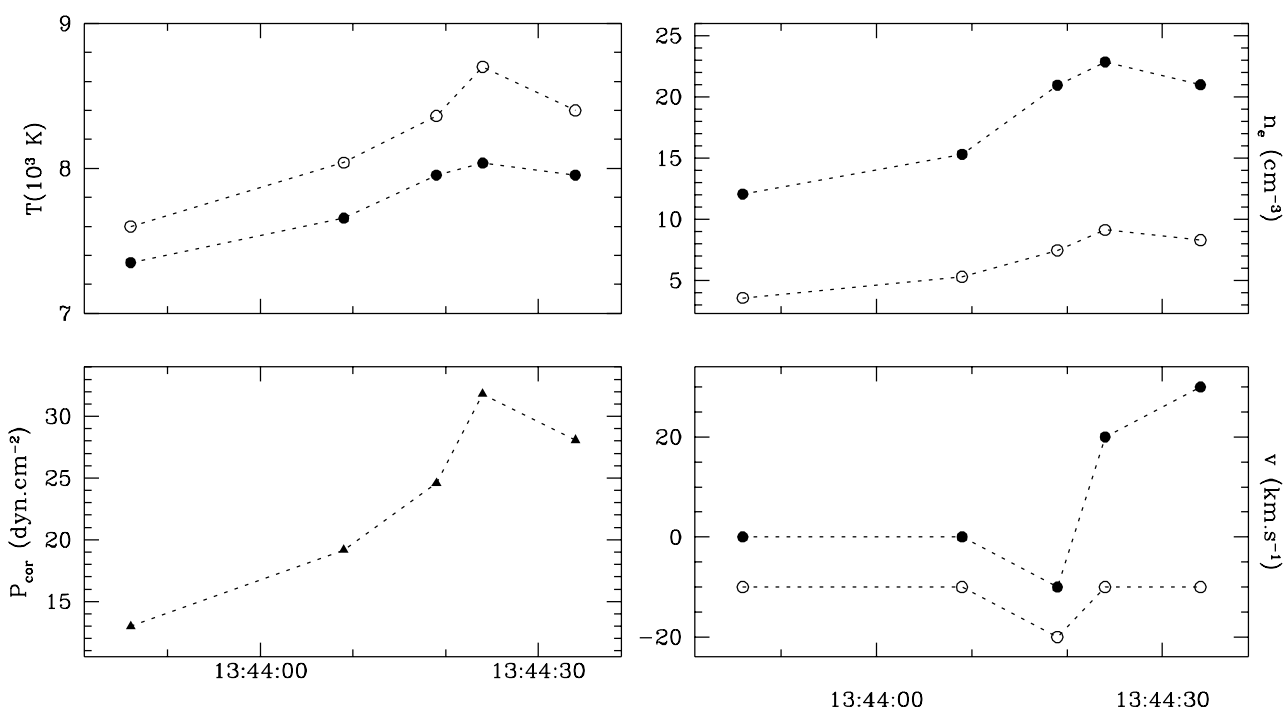

Fig. 8. Evolution with time of the temperature $T$, of the electron density $n_{\mathrm{e}}$, of the coronal pressure $P_{\text {cor }}$, and of the velocity $v$. Filled circles refer to the height $\approx 900$ and open circles to the height $\approx 1400 \mathrm{~km}$.

We find that in the high chromosphere the velocity is always directed upward, while in the lower layers it is directed upward at the beginning of the first spike, when the chromosphere undergoes a strong heating and/or compression, and within $6 \mathrm{~s}$ of the peak time of the spike the velocity becomes directed downward, and continues to increase even during the cooling phase.

The presence of an upward motion in the whole flaring atmosphere, before the settlement of the typical downward motion, might be a signature of the chromospheric evaporation, observed at chromospheric levels and not, as more common, at coronal levels. The upward motion could be the first dynamic chromospheric manifestation of a flare with a very short time response $(1 \mathrm{~s})$ with respect to the HXR spike. These arguments need a careful confirmation in the framework of models of the flare dynamics.

Two problems are raised by our models. The first is given by the fact that we cannot match, with the same temperature and velocity distributions, both the $\mathrm{Si}$ line profile and the far emission wings of $\mathrm{Ca} \mathrm{K}$ and $\mathrm{H}_{\delta}$ after the HXR burst. Since the emission wings of these lines are formed in the same region of the Si line, just above the temperature minimum, the velocity needed to reproduce the red wings observed at the times of models 4 and 5 would displace the Si line far beyond what is observed.

One possible explanation for this fact is that our underlying assumption of homogeneity in the analyzed area fails, and that the emission in the red wing is not due to velocities in the layers where the wing itself is formed, but it is due to a smaller kernel, below the resolution of the observations, with strong downward velocities at higher layers, where the line centers are formed. This suggests that the footpoints of the flaring loops have very small size, in the subarcsec regime, and supports the scenario of several very tiny loops differently involved in the flare.

The second problem is given by the presence of downward and upward velocities in the chromosphere at the same time, as found after the spike. One simple explanation might be that the observed line profiles are due to the merging in a small area of emission of adjacent regions with different velocities. This does not seem to be the case, since the seeing of our observations was very good, and the small area considered for modelling was embedded in a bigger area $\left(2^{\prime \prime} \times 6^{\prime \prime}\right)$ showing similar characteristics, although with lower velocity values. Another possibility is that the upward and downward velocities are the signatures of the chromospheric evaporation within a flaring loop. However, in this case one should be able to verify the upward and downward momentum balance. We computed such quantities and we found that the upward momentum of the high chromosphere is negligible if compared to the one of the high-density low chromosphere.

Hence, it seems difficult to explain all the flare characteristics with a single loop extending from the low chromosphere to coronal levels. We think probable that the line of sight intercepts different flaring loops with different dynamics, and that the upward velocity inferred in the high chromosphere at all times is due to a "second" loop always presenting an upward velocity. The presence of such a loop had indeed been inferred by CFFS on the basis of spectral measurements in areas adjacent to the flaring kernel.

Acknowledgements. We would like to thank the referee, Dr. Heinzel, for constructive comments that help us to improve the paper.

\section{References}

Abbett, W. P., \& Hawley, S. L. 1999, ApJ, 521, 906

Athay, R. G. 1976, in The Solar Chromosphere and Corona: Quiet Sun, (Reidel, Dordrecht, Ch. III)

Avrett, E. H., \& Loeser, R. 1984, in Methods in Radiative Transfer, ed. W. Kalkofen (Cambridge, Univ. Press), 341

Beckers, J. M. 1981, in The Sun as a Star, ed. S. Jordan, CNRS/NASA, 11 
Black, J. H., Weisheit, J. C., \& Laviana, E. 1972, ApJ, 177, 567

Brekke, P., Rottman, G. J., Fontenla, J., \& Judge, P. G. 1996, ApJ, 468, 418

Canfield, R. C., \& Gayley, K. G. 1987, ApJ, 322, 999

Canfield, R. C., Gunkler, T. A., \& Ricchiazzi, P. J. 1984, ApJ, 282,296

Canfield, R. C., Kiplinger, A. L., Penn, M. J., Wülser, J. P. 1990, ApJ, 363, 318

Cauzzi, G., Falchi, A., Falciani, et al. 1995, A\&A, 299, 611

Cauzzi, G., Falchi, A., Falciani, R., \& Smaldone, L. A. 1996, A\&A, 306, 625 - CFFS

Cincunegui, C. C., \& Mauas, P. J. D. 2001, ApJ, 552, 877

Ding, M. D., \& Fang, C. 1989, A\&A, 225, 204

Falchi, A. 2002, Il Nuovo Cimento C, in press

Falchi, A., Falciani, R., \& Smaldone, L. A. 1990, A\&AS, 84, 601

Falchi, A., Falciani, R., \& Smaldone, L. A. 1992, A\&A, 256, 255

Fang, C., Hénoux, J. P., \& Gan, W. Q. 1993, A\&A, 274, 917

Fisher, G. H. 1985, IAU Colloq. 89, Lect. Notes Phys., 255, 53

Fisher, G. H. 1989, ApJ, 346, 1019

Gan, W. Q., Fang, C., \& Zhang, H. Q. 1991, A\&A, 241, 618

Gan, W. Q., Rieger, E., \& Fang, C. 1993, ApJ, 416, 886
Giovanardi, C., Natta, A., \& Palla, F. 1987, A\&AS, 70, 269

Giovanardi, C., \& Palla, F. 1989, A\&AS, 79, 157

Gómez, D. O., \& Mauas, P. J. D. 1992, ApJ, 398, 682

Heinzel, P., Karlicky, M., Kotrč, P., \& Švestka, Z. 1994, Sol. Phys., 152, 393

Ichimoto, K., \& Kurokawa, K. 1984, Sol. Phys., 93, 105

Johnson, L. C. 1972, ApJ, 174, 227

Kasparová, J., \& Heinzel, P. 2002, A\&A, 382, 688

Konjevic, N., Dimitrijevic, M., \& Swiese, W. L. 1984, J. Phys. Chem. Ref. Data, 13, 649

Mathisen, R. 1984, Institute of Theoretical Astrophysics, Publication Ser., No. 1

Mauas, P. J. D. 1993, ApJ, 414, 928

Mauas, P. J. D., \& Falchi, A. 1994, A\&A, 281, 129

Mauas, P. J. D., \& Gómez, D. O. 1997, ApJ, 483, 496

Monteiro, et al. 1988, J. Phys B, 21, 4165

Scholz, T. T., et al. 1990, MNRAS, 242, 692

Shine, R. A., \& Linsky, J. L. 1974, Sol. Phys., 39, 49

Švestka, Z. 1976, in Solar Flares (Reidel Publishing Company)

Taylor, P. O., \& Dunn, G. H. 1973, Phys. Rev. A, 8, 2304

Vernazza, J. E., Avrett, E. H., \& Loeser, R. 1981, ApJS, 45, 635

Wülser, J. P., Canfield, R. C., Acton, L. W., et al. 1994, ApJ, 424,459 